\title{
Philonsorbonne
}

$14 \mid 2020$

Année 2019-2020

\section{Les usages de la sagesse divine en physique dans l'œuvre de G.W. Leibniz}

\section{Takuya HAYASHI}

\section{OpenEdition}

\section{$\checkmark$ Journals}

\section{Édition électronique}

URL : https://journals.openedition.org/philonsorbonne/1372

DOI : 10.4000/philonsorbonne.1372

ISSN : 2270-7336

Éditeur

Publications de la Sorbonne

\section{Édition imprimée}

Date de publication : 14 février 2020

Pagination : 25-45

ISSN : 1255-183X

\section{Référence électronique}

Takuya HAYASHI, «Les usages de la sagesse divine en physique dans l'œuvre de G.W. Leibniz »,

Philonsorbonne [En ligne], 14 | 2020, mis en ligne le 25 février 2020, consulté le 08 juin 2021. URL

http://journals.openedition.org/philonsorbonne/1372; DOI : https://doi.org/10.4000/philonsorbonne. 1372

(c) Tous droits réservés 


\title{
Les usages de la sagesse divine en physique dans l'œuvre de G.W. Leibniz
}

\author{
Takuya HAYASHI
}

\section{Introduction}

Le présent article essaie de retracer les évolutions de l'idée leibnizienne de sagesse divine en particulier dans la physique afin d'éclaircir le rapport qu'elle entretient avec la conception de la métaphysique. Pour rappeler l'idée de la sagesse divine dans la période de la maturité, le célèbre paragraphe 225 des Essais de Théodicée $e^{1}$ mérite d'être évoqué d'emblée, puisque ce passage très riche contient plusieurs éléments que nous abordons dans cet article. En nous référant donc à ce passage, et à d'autres textes qu'il serait plus difficile d'évoquer dans leur intégralité dans un article essentiellement consacré à la physique, nous proposons de distinguer les principaux éléments concernant la sagesse. Bien qu'ils ne couvrent pas toutes les caractérisations de la sagesse dans la pensée de Leibniz et que leurs distinctions ne soient pas absolues, on pourrait en repérer huit par commodité ${ }^{2}$.

1/ À la différence des sagesses finies des autres êtres, y compris les anges, seule la sagesse de Dieu, qui comprend une infinité de possibles et fait des combinaisons et des réflexions sur eux, est infinie, voire «plus qu'infinie ${ }^{3}$ ». En raison de cette immensité, elle s'appelle aussi omniscience ${ }^{4}$.

1. GP VI, 252. - Nous citons les textes de Leibniz selon les sigles suivants: GP = Die philosophische Schriften, éd. C. I. Gerhardt, Hildesheim, Olms, 1965; A = Sämtliche Schriften und Briefe, Darmstadt (Berlin), 1923-; Grua = Textes inédits, d'après les manuscrits de la Bibliothèque provinciale de Hanovre, publiés et annotés par Gaston Grua (2 tomes), Paris, PUF, 1948 ; réimpression : PUF, « Épiméthée », 1998.

2. Notre but n'est pas de faire le commentaire de ce passage de la Théodicée.

3. A II, 2, 515.

4. Causa Dei, § 13, GP, VI, 440. 
2/ Les degrés de perfection des possibles sont indépendants de sa volonté et estimés par sa sagesse. En ce sens, la sagesse est «la science de la bonté (scientia boni $)^{5} »$.

3/ Cette sagesse forme non seulement des combinaisons finies, mais aussi une infinité de mondes possibles ou de systèmes universels dont chacun a son propre ordre général.

4/ Chaque monde possible contient une infinité de possibles. Bien que la question reste en suspens de savoir si cela vaut pour tous les mondes possibles, l'important est qu'à la différence des possibles considérés à part, le monde qui sera choisi contient une infinité de créatures possibles.

5/ Avant toute volonté d'existence, elle envisage et compare une infinité de mondes possibles et trouve infailliblement le meilleur vrai. « ... la sagesse ou la connaissance du vrai est la perfection de l'entendement ${ }^{6} »$.

6/ Le choix se fait donc parmi les mondes possibles et non parmi les possibles considérés à part.

7/ Comme le meilleur plaît à la sagesse, ce qui est choisi est le meilleur.

8/ Ses opérations sont éternelles et se font ensemble. En ce sens aussi, la sagesse de Dieu se qualifie comme parfaite ${ }^{7}$.

Toutes ces opérations appartiennent à la même sagesse. Cependant, il semble qu'il vaille mieux les distinguer les unes des autres, et cela pour trois raisons : - Tout d'abord, dans la carrière de Leibniz, certaines d'entre elles ne sont mises en scène que relativement tard, ce qui justifie la méthode génétique de l'enquête. - Ensuite, la plupart de ces opérations sont conçues exclusivement comme celles de l'entendement (1-5), bien que certaines soient aussi liées à la volonté créatrice choisissant le meilleur, en tout cas pour ce qui regarde la question de l'existence du monde $(6,7)$. Les possibles et leurs perfections sont les objets nécessaires de la sagesse entendue comme scientia boni, mais ils ne dépendent pas de la sagesse considérée comme principe de l'existence ou volonté choisissant le plus parfait ${ }^{8}$. En négligeant cette distinction, on risque donc de confondre l'entendement avec la volonté. - Enfin, à chaque fois que Leibniz se réfère à la sagesse divine, il mobilise un ou plusieurs aspects sans penser à tous ensemble.

Il nous semble qu'il s'agit d'une notion relativement méconnue malgré son importance. Bien entendu, on sait très bien que la sagesse est l'attribut principal du Dieu de Leibniz ${ }^{9}$. Peu de commentaires sur sa métaphysique ne la mentionnent pas. À notre connaissance, toutefois, les recherches qui la

5. À Fabri, 1677, A III, 2, 145.

6. Causa Dei, § 18, GP, VI, 441.

7. GP VI, 399.

8. Cf. À Bourguet, GP III, 592 : «Les idées ou essences sont toutes fondées sur une necessité independante de la sagesse, de la convenance et du choix; mais les existences en dependent »; A VI, 4, 2008 : divina voluntate sive sapientia.

9. Cf. À Christian Philipp: «Dieu estant la sagesse même ne manque pas de choisir le meilleur » (A II, 1, 788). 
thématisent ne sont pas si nombreuses en proportion de son poids. Les études antérieures peuvent ainsi être classifiées en fonction de trois contextes majeurs.

Le premier est celui de la jurisprudence et de l'apologétique. La définition de la justice comme charité du sage, acquise depuis l'époque de Mayence, vaut pour Dieu et pour les hommes univoquement. Et la thèse selon laquelle Dieu a créé le meilleur des mondes possibles par sa sagesse, bonté et puissance est centrale dans la Théodicée. Cette question est abordée de façon systématique par Paul Rateau dans ses ouvrages ${ }^{10}$. Et c'est précisément dans ce contexte que Christiane Frémont développe ses différents arguments dans son article «Leibniz ou la sagesse de Dieu » ${ }^{11}$.

Le second contexte concerne plutôt la physique. Récemment, Daniel Garber a ouvert le débat au chapitre 6 de son ouvrage, consacré à «la sagesse divine et les causes finales ${ }^{12}$, ainsi que dans un article ${ }^{13}$. Il soutient qu'un grand changement intervient en 1678 ou 1679, lorsque Leibniz commence à fonder le principe de la mécanique sur la sagesse de Dieu, probablement à la suite de sa confrontation avec Spinoza. Mogens Laerke trouve l'engagement décisif à la sagesse dans les notes sur l'Ethique ${ }^{14}$. En reprenant la question dans son article, Osvaldo Ottaviani affirme que, dans sa discussion avec N. Sténon datée de la fin 1677, Leibniz abandonne complètement la position quasi-nécessitariste au profit de la version inchoative de sa doctrine des mondes possibles ${ }^{15}$. Ansgar Lyssy ${ }^{16}$ examine la notion d'économie divine dans l'œuvre de Leibniz allant des premiers textes physiques jusqu'aux textes tardifs, dans lesquels des caractères religieux et moraux de cette notion sont mis en valeur.

Le troisième contexte, enfin, examine le rapport entre la sagesse et la recherche des raisons par le principe de raison. On a l'habitude d'évoquer le passage suivant : " Je commence en philosophe, mais je finis en théologien. Un de mes grands principes est que rien ne se fait sans raison. C'est un principe de philosophie. Cependant, dans le fonds ce n'est pas autre chose que l'aveu de la sagesse divine, quoique je n'en parle pas d'abord ${ }^{17} »$.

10. P. Rateau, La question du mal chez Leibniz : fondements et élaboration de la Théodicée, Paris, Honoré Champion, 2008 ; Leibniz et le meilleur des mondes possibles, Paris, 2015.

11. Ch. Frémont, «Leibniz ou la sagesse de Dieu », in Luc Langlois et al., Les philosophes et la question de Dieu, Paris, Presses Universitaires de France, coll. «Fondements de la politique », 2006, p. 131-145.

12. D. Garber, Leibniz : Body, Substance, Monad, Oxford UP, 2009.

13. Id., "Leibniz, Theology and the Mechanical Philosophy», Branching Off, 2009, p. 167-186.

14. M. Laerke, «Leibniz on the Principle of Equipollence and Spinoza's Causal Axiom », The Leibniz Review, Vol. 25, 2015, p. 123-130.

15. O. Ottaviani, «Divine Wisdom and Possible Worlds. Leibniz's Notes to the SpinozaOldenburg Correspondence and the Development of His Metaphysics », in Studia Leibnitiana, Bd. 48, H. 1 (2016), p. 15-41.

16. A. Lyssi, «Nature and Grace: On the Concept of Divine Economy in Leibniz's Philosophy », in Studia Leibnitiana, Bd. 48, H. 2 (2016), p. 151-177.

17. E. Bodemann, Die Leibniz-Handschriften, Hannover, 1889, reprint Olms, 1966, p. 58. 
Michaël Devaux reprend l'analyse de Vincent Carraud $^{18}$ et soutient que «le principe de raison suffisante n'est exercé que par Dieu, l'homme ne peut rendre que des raisons déterminantes ${ }^{19} \gg$. Tout en reconnaissant les évolutions des principes de raison, Arnaud Lalanne situe, quant à lui, la Raison divine ou la sagesse dans "le centre ou le point de convergence ${ }^{20}$ dans la philosophie de Leibniz.

Ce dernier point semble diminuer, cependant, l'importance de la question de la sagesse, car cette question paraît se réduire à celle du principe de raison et à celle de l'harmonie. Comme Leibniz parle de «la sagesse de l'auteur des choses qui ne fait rien sans harmonie et sans raison ${ }^{21} »$, on pensera que la notion de la sagesse n'est que subsidiaire. C'est bien le cas sans doute, et cela pourrait expliquer pourquoi on ne l'a pas assez thématisée. Néanmoins, nous bornant ici au deuxième contexte qui aborde la relation entre physique et métaphysique, nous tenterons de montrer l'importance de la sagesse en repérant, par une enquête centrée sur le terme de sagesse divine, les diverses manières de l'utiliser que Leibniz élabore durant sa vie. - Dans un premier temps donc, abordant la période de la réforme de la physique vers la fin des années 1670 , nous soutiendrons que cette réforme entraîne celle de la théologie naturelle de même qu'elle produit une nouvelle «métaphysique» qui sera désormais dominante dans son œuvre. - Dans un second temps, nous passerons à la seconde moitié des années 1680 et examinerons aussi bien une nouvelle façon d'employer la sagesse au moyen du principe de l'ordre général qu'un argument sur certaines propriétés de la sagesse de Dieu qui conduit à l'unicité du décret divin. - Dans un troisième temps, nous analyserons d'autres manières intervenues vers la seconde moitié des années 1690, lors de la naissance de l'hypothèse de l'harmonie préétablie ainsi que de l'idée de machine de la nature, et nous montrerons à ce titre un changement subtil survenu au sujet de la relation entre mathématiques, physique et métaphysique. - Enfin, nous conclurons en suggérant le motif pour lequel Leibniz s'est attaché à connaître la sagesse divine.

\section{La période avant $\mathbf{1 6 8 0}$}

Un changement qui s'est opéré dans la sagesse divine est suggéré par Joseph Moreau. Selon lui, dans l'Hypothesis physica nova de 1671, les lois de la nature étaient encore étrangères et extérieures à la sagesse divine. " La finalité était [...] une utilisation par l'intelligence des lois nécessaires et

18. V. Carraud, Causa sive ratio, Paris, PUF, 2002, p. 417-427.

19. M. Devaux, «Le fondement de la métaphysique réelle chez Leibniz », Philosophie 2015/4 $\left(\mathrm{n}^{\circ} 127\right)$, p. $70-83$, ici p. 81.

20. A. Lalanne, Genèse et évolution du principe de raison suffisante dans l'œuvre de Leibniz, 2013, p. 195.

21. Nouveaux Essais sur l'entendement humain, GP V, 49. 
aveugles du mouvement ${ }^{22} \gg$ qui étaient indépendants de lui. En revanche, dans les textes de la maturité, les lois de la nature ont leur origine dans la sagesse de Dieu et expriment une nécessité morale ou nécessité de choix. La finalité n'est plus donc «l'utilisation par l'intelligence d'un mécanisme extérieur à elle ; elle est principe même du mécanisme » (Ibid. p. 166). Plus récemment, en analysant les textes portant sur le principe d'équipollence entre la cause pleine et l'effet entier, D. Garber ${ }^{23}$ a fait remarquer un changement subtil de son statut. Il a montré qu'en abandonnant sa tentative initiale pour le démontrer à partir des définitions par une nécessité géométrique $^{24}$, Leibniz est parvenu vers 1678 ou 1679 à soutenir que le principe dépend de la sagesse divine ${ }^{25}$. Selon lui, pour Leibniz, qui vise à concilier mécanisme et piété, l'échec de sa tentative initiale était sans doute bienvenu. Il estime que le bannissement des causes finales dans l'Éthique de Spinoza a occasionné leur réintroduction dans la physique chez Leibniz. De la sorte, la sagesse divine devient importante dès la fin des années 1670 aussi bien comme origine du principe de l'équipollence que comme origine des lois de la nature qui en résultent. En revanche, chez le jeune Leibniz, Dieu était requis comme chez Descartes en tant que cause efficiente qui explique certains phénomènes ${ }^{26}$. Certes, Leibniz mentionne la sagesse divine dans certains textes comme la Confessio naturae contra atheistas publiée en $1669^{27}$, ou encore les paragraphes $46^{28}$ et $51^{29}$ de $1^{\prime}$ Hypothesis physica nova. Suivant la lecture de Daniel Garber, toutefois, la sagesse divine dans la Confessio naturae est "subordonnée à Dieu en tant que cause efficiente de figure, mouvement et consistance» (op. cit., p. 13). De même, le recours à cet attribut comme principe de parcimonie dans l'Hypothesis physica semble

22. J. Moreau, L'Univers leibnizien, Paris-Lyon, 1956, p. 164.

23. Op. cit.

24. Voir entre autres De arcanis motus et mechanica ad puram geometriam reducenda (février-septembre 1676), A VIII, 2, 135.

25. Voir par exemple une lettre à Christian Philipp, décembre 1679, A II, 1, 767.

26. D. Garber, art. cit., p. 167.

27. «Or pourquoi l'être incorporel choisirait-il cette grandeur, cette figure et ce mouvement plutôt que d'autres, on ne peut en rendre raison, s'il n'est intelligent et sage puisque les choses sont belles, puissant puisqu'elles obéissent au moindre signe. Et donc un tel être incorporel sera l'Esprit recteur du monde entier, c'est-à-dire DIEU. » (A VI, 1, 492, tr. par Ch. Fremont, Leibniz. Discours de métaphysique et autres textes, Paris, Garnier-Flammarion, « GF », 2001, p. 27).

28. «... alors que pourtant la plupart du temps les expériences, comme j'ai montré pour le mouvement, diffèrent tout à fait à première vue des principes intimes de la réalité et ne se concilient avec eux que par la grande ingéniosité de l'économie universelle, par l'admirable sagesse du créateur qui englobe les origines des choses. » (GP IV, 202, tr. par René Violette, Leibniz. Physique et métaphysique. Opuscules de jeunesse, Limoges, Lambert-Lucas, 2012, p. 113).

29. «J'ai toujours cru, cependant, que l'admirable sagesse du Créateur a ménagé les choses de telle façon que beaucoup soit régi par peu. » (GP IV, 204, tr. par René Violette, op. cit., p. 114). Cf. $\S 59$ : Atque hie admirari licet praxin DEI in oeconomia rerum geometrisantis (GP IV, 216). 
être « une pensée après coup » (Ibid. p. 22) pour reprendre les paroles de ce commentateur ${ }^{30}$.

Nous partageons globalement cette ligne de lecture ${ }^{31}$. De fait, malgré l'existence de plusieurs premiers textes qui font mention de la sagesse de Dieu, une autobiographie tirée des Nouveaux Essais sur l'entendement humain montre que le jeune Leibniz ne l'avait pas reconnue et qu'il ignorait la recherche des causes finales :

« Vous savés que j'estois allé un peu trop loin ailleurs et que je commençois à pencher du costé des Spinosistes, qui ne laissent qu'une puissance infinie à Dieu, sans reconnoistre ny perfection ny sagesse à son egard, et méprisant la recherche des causes finales dérivent tout d'une necessité brute. Mais ces nouvelles lumières m'en ont gueri; et depuis ce temps là je prends quelquesfois le nom de Théophile ${ }^{32}$. »

Selon Paul Rateau, dans un article intitulé « La nécessité de l'optimum dans la Confessio philosophi : un nécessitarisme leibnizien ? ${ }^{33}$, Leibniz se démarque du nécessitarisme strict adopté dans la Confessio philosophi par la reconsidération du rôle de la volonté divine ainsi que par l'approfondissement de la notion de possible. Ce commentateur repère trois éléments, à savoir l'extériorité de Dieu par rapport à la série des choses, une nécessité morale et une conception forte du possible doté de réalité et même d'une tendance à l'existence. Nous appuyant sur ce résultat, nous voudrions ajouter à l'enquête menée par D. Garber trois points à propos de la sagesse divine.

\section{1/ Concernant les premiers textes}

Pour mieux penser la mise en valeur de la sagesse de Dieu depuis la fin des années 1670, examinons rapidement quelques autres textes de jeunesse. La lettre à Mariotte datée du juillet 1673 semble confirmer que la sagesse divine n'était pas encore établie comme principe métaphysique de la physique. Leibniz reproche à Descartes d'avoir recours à un principe faible de sagesse divine :

30. François Duchesneau donne une interprétation similaire (F. Duchesneau, La Dynamique de Leibniz, Vrin, 1994, p. 203).

31. Claire Schwarz, tout en disant justement qu'« on pourrait même considérer que la Confessio naturae identifie Dieu comme cause non seulement efficiente et formelle mais également finale de l'univers ", rejette avec D. Garber l'idée de lire ce texte à l'aune des textes de la maturité. (C. Schwarz, Leibniz. La raison de l'être, Paris, Belin, 2017). Une analyse détaillée du texte est donnée par P. Rateau, «Les preuves leibniziennes de l'existence de Dieu : la "voie" du mouvement », Les Études philosophiques, 2016/3 (n 163), p. 357-386.

32. A VI, 6, 73. Cf. A VI, 4, 1653 ; Dialogue entre Poliandre et Theophile (A VI, 4, 2220 2227, sans doute 1679).

33. P. Rateau, «La nécessité de l'optimum dans la Confessio Philosophi : un nécessitarisme leibnizien? », dans Raphaële Andrault, Mogens Lærke, Pierre-François Moreau (dir.), Spinoza/Leibniz. Rencontres, controverses, réceptions, Paris, Presses universitaires de ParisSorbonne, 2014, p. 161-173. 
« Je me rendis facilement à ces considerations, d'autant plus que ce que Monsieur des $\mathrm{Cartes}^{34}$ avoit dit touchant la reflexion des corps ne pouvoit pas asseurément, passer pour demonstré, par ce que sa supposition, que le mouvement ne se perd pas, est appuyée sur un principe bien foible, et peu digne d'un si grand homme comme luy, sçavoir que la sagesse de Dieu est interesseée à ne laisser rien perdre dans le monde. » (A II, 1, 371)

On pourra se demander pourquoi Leibniz dénonce le recours cartésien à la sagesse divine. En réalité, Descartes utilisait le terme immutabilitas, comme Leibniz le reconnaissait lui-même dans sa lettre à Ordenburg datée du 25 octobre $1671^{35}$. Notre but n'est pas d'examiner la confrontation des deux philosophes. Nous nous contentons ici de remarquer deux choses que ces deux textes semblent nous suggérer : tout d'abord, en 1673, Leibniz n'établissait pas encore la sagesse divine comme son propre principe. Elle était même attribuée au Dieu de Descartes, quoique Leibniz distinguait un recours mal-fondé à la sagesse divine de celui fondé sur la nature du ressort ${ }^{36}$. Ensuite, cet attribut n'était pas encore lié à la considération de la convenance ou du meilleur parmi une infinité de possibles. On comprendra mieux ces textes en les comparant avec un passage de 1692. Pour critiquer Descartes, Leibniz y emploie désormais le terme constantia Dei, mais c'est en y opposant la sapientia de son propre Dieu :

Nam quae a Cartesio affertur de constantia Dei, infirma admodum est, neque enim is qui constans est, eandem semper cujusque rei quantitatem servat, alioqui sequeretur, eundem semper esse numerum hominum, imo muscarum. Sufficit eum certa quadam lege uti, sed quae ex tot legibus aptissima sit et servatu dignissima, non ex constantia Dei, sed ex sapientia ejus definiendum est. Sapientis est constantem in optimis, in mediocribus varium esse ${ }^{37}$.

Cela n'empêche pas que le jeune Leibniz reconnaissait un certain rôle organisateur de la sagesse en physique. Comme A. Lyssy le soutient, le fait que les phénomènes de la nature convergent avec les principes abstraits qui

34. Cf. Discours de la méthode, La dioptrique, disc. II, dans l'édition Adam-Tannery des Euvres complètes de Descartes, 11 vol., Paris, Vrin-CNRS, 1964-1974 (désormais noté : AT), AT VI, 93-105 ; Principia philosophiae, II, 36 (AT VIII, 1, 61).

35. Cartesius iterum recurrit ad immutabilitatem, et regularitatem Dei (A II, 1, 272).

36. Dans la lettre à Mariotte citée au-dessus, Leibniz continuait en effet : "S'il avoit songé à la nature du ressort, il ne se seroit pas servi de ce sacré refuge d'un miracle perpetuel » (A II, 1, 371). Sur la position de Descartes, voir L. Devillairs, « L'immutabilité divine comme fondement des lois de la nature chez Descartes et les éléments de la critique leibnizienne », in Revue d'histoire des sciences, tome 54, $\mathrm{n}^{\circ} 3,2001$. p. 303-324.

37. A III, 5, 441. Ajoutons que ce texte diffère du Pacidius Philalethi de 1676, qui tout en soulignant l'attribut de sagesse, l'identifiait avec la constance opérante dans ce qui s'appelait la «transcréation » sans assez considérer les forces inhérentes du corps, ni l'aptitude ou la convenance des lois que la sagesse garde, ni l'unicité du décret divin tel que nous le traiterons ci-dessous. 
les gouvernent ne peut s'expliquer logiquement par aucune science, puisque cela concerne la création du monde. Pour l'expliquer il faut recourir à une «bienfaisance incrédible ${ }^{38}$ » de Dieu-géomètre ${ }^{39}$. Il convient d'ajouter entre autres une lettre à J. Thomasius de 1670. Après avoir invoqué un passage du Phèdre de Platon où Socrate est déçu par Anaxagore, qui, bien qu'il ait posé les deux principes que sont la matière et l'esprit, a fini par se rapprocher de la pensée matérialiste de Démocrite, Leibniz poursuit :

« On trouve la même pensée chez les physiciens modernes, qui en quête des causes matérielles, négligent les causes rationnelles de la réalité, alors que si la sagesse du créateur resplendit quelque part de tous ses feux, c'est bien dans le fait d'avoir réglé l'horloge du monde [horologium mundi] de telle sorte que tout, par la suite, comme sous l'effet d'une certaine nécessité, conspire à la plus haute harmonie de l'ensemble... Or l'esprit premier, en raison de sa sagesse, a institué les choses, au départ, de telle sorte qu'il ne soit pas nécessaire, pour leur conservation, de compter sur la facilité de son concours extraordinaire. C'est ainsi que personne n'irait faire l'éloge de ce fabriquant d'automates qui serait contraint de corriger tous les jours quelque chose à son propre ouvrage $»^{40}$.

On voit ici l'opposition entre un Dieu sage et un Dieu similaire à l'homme qui corrige toujours son ouvrage. L'idée de l'horloge du monde ${ }^{41}$ parfaitement réglée au départ est semblable à l'image d'une montre figurant dans la période de maturité. On pourrait aller jusqu'à dire que la controverse avec Clarke hérite et approfondit cette problématique ${ }^{42}$. Pourtant, bien que le jeune philosophe parle de la plus haute harmonie de l'ensemble, il ne la fonde pas sur la considération des possibles inexistants et il ne considère pas non plus la série des choses sub ratione possibilitatis. De plus, ne se trouve pas encore l'affirmation selon laquelle il faut employer la sagesse divine dans la physique, et encore moins les réflexions sur les manières dont on le fait et sur les conditions de cet emploi.

\section{2/ La réforme de la physique est relative à la réforme de la théologie naturelle}

On sait que Leibniz a rédigé le résumé latin du Phèdre en mars ou avril 1676 (A VI, 3, N. 20). Et sans doute à travers sa lecture de l'Éthique de Spinoza et des Principes de la philosophie de Descartes, il se livre aux réflexions sur les choses qu'il ne considérait pas. Parmi ceux qu'il appelle

38. Hypothesis physica nova, § 59, GP IV, 216.

39. Sur le détail, voir A. Lyssy, art. cit., p. 160.

40. GP I, 33 ; A II, 1, 119, tr. par R. Bodeus, in Leibniz-Thomasius. Correspondance 16631672, Paris, Vrin, 1993, p. 277.

41. Cette idée apparaissait dans une lettre précédente à Thomasius (30 avril 1669, A II, 1, 35).

42. Cf. GP VII, 352. 
« deux sectes de Naturalistes » vers 1679 à $1680^{43}$, la secte des épicuriens faisait bien l'objet de critique dans la lettre à Thomasius, tandis que la secte des stoïciens n'était pas encore condamnée, au moins explicitement. Or c'est à celle-ci que Leibniz associe la philosophie nécessitariste de Spinoza, et même celle de Descartes en raison de quelques thèses risquant de détruire indirectement la sagesse divine. Aux yeux de Leibniz, c'est maintenant Descartes qui s'approche le plus d'Anaxagore et qui, tout en établissant l'intelligence comme un principe, ne l'a pas assez utilisée. Sans doute afin de s'écarter des thèses cartésiennes et de concilier raison et piété, il s'interroge de nouveau sur l'idée de Dieu, en particulier celle de sagesse. Il ne suffit plus d'affirmer que Dieu est un sage qui a réglé l'horloge du monde. Il faut rejeter le nécessitarisme absolu, incompatible avec la sagesse et les thèses qui pourraient y conduire, comme la thèse selon laquelle la matière reçoit toutes les formes successivement. C'est ainsi que Leibniz commence à soutenir qu'il y a les possibles inexistants, qui ne s'actualisent jamais, mais qui demeurent possibles et existent dans l'entendement divin. De fait, ceux qui font l'objet de critiques dans le Quod non omnia possibilia ad existentiam parveniant (A VI, 4, 1352), sans doute rédigé en 1677, ce sont les Dieux de Descartes et de Spinoza. C'est à cette période que Leibniz commence à soutenir qu'on doit employer utilement la sagesse divine et les causes finales en physique, non seulement pour admirer la sagesse divine, mais aussi pour découvrir et manier les vérités difficiles à découvrir par la recherche des causes efficientes comme les vérités de l'optique ${ }^{44}$.

Il est intéressant de noter que même la conduite de Dieu commence à s'expliquer à l'image de la manière d'agir de la nature suivant les causes finales. L'ébauche de cette tentative se trouve dans la Demonstratio quod Deus omnia possibilia intelligit ${ }^{45}$ de 1677 , qui utilise un exemple de "quelque liquide compressé qui s'efforce de sortir». Il convient de souligner que l'apparition de la doctrine de la tendance ou du conatus des possibles à l'existence confirmera cette orientation. On peut certes l'entendre dans la continuité des écrits précédents comme le Principium meum ${ }^{46}$. Pour autant, Leibniz semble franchir un grand pas en décrivant à l'image de la nature la conduite de Dieu suivant le principe de détermination. On peut sans doute mieux comprendre son intention si l'on situe cela dans son projet d'employer utilement la sagesse divine en physique. Afin de bien l'utiliser en physique non seulement en général comme la raison dernière des lois de la nature, mais aussi en particulier pour les découvertes des vérités, il faudrait que la manière d'agir de l'auteur de la nature soit en correspondance structurelle avec celle de la nature qui a pour but de prendre la voie la plus

43. A VI, 4, 263.

44. Cf. À Christian Philippe, décembre 1678, A II, 1, 767. Voir aussi la lettre à Malebranche du 23 janvier 1679 (A II, 1, 678). Leibniz y apprécie « un tres bel usage des causes finales » fait par Malebranche.

45. A VI, 4, 1353, tr. par M. Laerke, Leibniz. Lecteur de Spinoza, Paris, 2008, p. 802. 46. A VI, 3, 581f. 
facile et déterminée. Ainsi, le changement qui a eu lieu en physique n'a pas manqué d'entraîner le changement en théologie naturelle.

\section{3/ La théologie naturelle ainsi réformée s'approprie le nom de métaphysique}

Il n'est pas sans intérêt de noter que la métaphysique dont le principe de l'équivalence dépendait initialement n'est pas la même que la métaphysique dont ce principe dépend désormais. Celle-là était conçue comme ce qui démontre tant ce principe de la mécanique que celui de la géométrie. Une telle conception de la métaphysique que l'on pourrait peut-être associer à l'Ontologia semble perdurer jusqu'à la fin des années $1670^{47}$. Au contraire, si le nouveau principe de l'équivalence dépend de la métaphysique ou s'il est lui-même le principe métaphysique, c'est dans la mesure où il a son origine dans la sagesse de Dieu, c'est-à-dire dans sa libre volonté choisissant le plus parfait :

Quodsi scivissent illi qui leges mechanicas oppugnant, ipsas leges mechanicas denique resolvi in rationes metaphysicas, et rationes illas metaphysicas a divina voluntate sive sapientia oriri non adeo mechanicas explicationes aversarentur ${ }^{48}$.

Cette métaphysique que l'on pourrait appeler métaphysique de la sagesse divine, liée à la réhabilitation des formes substantielles ${ }^{49}$ constituera désormais ce que Leibniz désigne en premier sens par le terme de métaphysique, même si les dispositifs conceptuels évolueront ultérieurement.

N'est-elle pas présente en un sens dès la première période ? Dans son article récent, "Philosophy and Science in Leibniz», M.R. Antognazza a soutenu que le rapport entre physique et métaphysique est constant dès la Confessio Naturae, tout en admettant que les réflexions sur le principe incorporel y étaient insuffisantes :

47. « Sed principia metaphysica sunt communia divinis et humanis, quia agunt de Veritate et de Ente in genere, quod est commune Deo et creaturis. » (Dialogus inter theologum et misosophum [seconde moitié 1678 à première moitié 1679 (?)], A VI, 4, 2215).

48. Praefatio ad libellum elementorum physicae [été 1678 à hiver 1678/79 (?)], A VI, 4, 2008. Cf. Principium mechanicae universae novum, E. Bodemann, Leibniz Handschriften (manuscrits) : Niedersächsische Landesbibliothek, Hanover, XXXV, 10, 5, f 3r, trad. par M. Fichant, op. cit., p. 287, 1680-1688 selon M. Fichant.

49. Cf. cette remarque de Michel Fichant: «Il est donc clair qu'à ce stade d'élaboration la constitution d'une nouvelle mécanique fondée sur la loi de conservation de la force $(m v)$ suggère une nouvelle métaphysique où l'univers est partout peuplé de formes ou d'âmes dotées de perception et d'appétition, parmi lesquelles ne sont des esprits (mentes) que celles qui ont des concepts distincts et sont en commerce avec Dieu.» (M. Fichant, "Mécanisme et métaphysique: le rétablissement des formes substantielles (1679) », in Science et métaphysique dans Descartes et Leibniz, Paris, PUF, 1998, p. 197). 
As regards the conception of the relationship between physics and metaphysics, it seems to me that Leibniz remains committed to the same position as the Confessio Naturae: metaphysical principles - whether conceived as substantial forms or as some other incorporeal principle - ought not to enter into physical explanations of natural phenomena. Although at the time of the Confessio Naturae he had not yet developed the tools for thinking in a satisfactory way about the incorporeal principle required by bodies, he had already matured a view on the relationship between physics and metaphysics which later breakthroughs in both fields did not change ${ }^{50}$.

Il est vrai qu'il y a une certaine continuité. La métaphysique de la sagesse divine n'est pas une création ex nihilo. Cependant, nous croyons que les caractérisations du principe incorporel sont essentielles pour penser le rapport entre physique et métaphysique. Par ailleurs, le terme de métaphysique ne figure pas dans la Confessio naturae. De plus, ce n'est sans doute pas un terme privilégié et bien établi pour désigner la science des choses immatérielles, comme dans les textes de la maturité, même si Leibniz suit une tradition qui conçoit la métaphysique comme théologie naturelle. En fait, ce n'est que vers la fin des années 1670 qu'une conception de la métaphysique liée à la sagesse divine apparait en tant que telle et constitue une métaphysique dominante dans son œuvre ${ }^{51}$. En se rapportant à la nouvelle science de la dynamique, cette métaphysique portera le nom de « métaphysique réelle » à partir des années 1690 .

\section{La seconde moitié des années 1680 : l’ordre général et le principe de continuité}

Cet acquis de la fin des années 1670 demeurera important durant le reste de la vie du philosophe de Hanovre, comme en témoignent par exemple le Tentamen Anagogicum : Essay anagogique dans la recherche des causes ${ }^{52}$, une lettre à Christian Wolff ${ }^{53}$ ou encore le paragraphe 11 des Principes de la nature et de la grâce $e^{54}$. Dans le Discours de métaphysique aussi, il répète la

\footnotetext{
50. M. R. Antognazza, «Philosophy and Science in Leibniz », in L. Strickland, E. Vynckier \& J. Weckend (eds.), Tercentenary Essays on the Philosophy \& Science of G.W. Leibniz, Palgrave-Macmillan. 2016, p. 29.
}

51. Nous ne pouvons pas traiter ici de tous les textes concernant ce sujet. En particulier l'examen de la lettre vraisemblablement adressée à Elisabeth (novembre 1678) sera requis ailleurs.

52. «Elles [les lois du mouvement] ont leur origine dans la sagesse de l'auteur, ou dans le principe de la plus grande perfection, qui les a fait choisir. » (GP VII, 271) Cela constitue d'ailleurs une preuve la plus efficace de Dieu (GP VII, 279).

53. 1710 et 1711. Briefwechsel zwischen Leibniz und Christian Wolff, ed. by C.I. Gerhardt, Halle, H.W. Schmidt, 1860, p. 129.

54. GP VI, 605. 
nécessité de l'usage des causes finales et de la sagesse de Dieu dans la physique. Mais, dans la seconde moitié des années 1680, deux nouvelles considérations de la sagesse divine se trouvent thématisées. L'une est exposée dans un article bien connu de 1687 ; l'autre se développe dans des lettres au landgrave Ernest de Hesse-Rheinfels et à Antoine Arnaud.

L'intention de bien employer la sagesse divine pour les explications en physique est soulignée par le titre lui-même d'un article, «Lettre de M. L. sur un principe general utile à l'explication des loix de la nature par la consideration de la sagesse divine, pour servir de replique à la reponse du R. P. D. Malebranche ». Il convient de noter que le «principe de l'ordre général » ou celui de continuité ${ }^{55}$ est ici assimilé à ce qu'on peut utiliser pour expliquer les lois de la nature par la sagesse divine. Autrement dit, ce principe sert de moyen utile lorsque l'on explique les lois de la nature en considérant la sagesse divine. Selon Leibniz, « Il a son origine de l'infini, il est absolument necessaire dans la Geometrie ${ }^{56}$, mais il reussit encor dans la physique » (GP III, 52). Pourquoi ? Précisément «par ce que la souveraine sagesse, qui est la source de toutes choses, agit en parfait geometre, et suivant une harmonie à laquelle rien ne se peut adjouter» (Ibid.). Ce que Leibniz veut dire ici par l'expression de parfait géomètre n'est sans doute pas le fait que Dieu agit de manière déterminée ${ }^{57}$, mais plutôt le fait que Dieu se règle sur le principe d'origine mathématique. Rappelons

55. Du principe général selon lequel «Datis ordinatis, etiam quaesita sunt ordinata», dépendent deux variantes suivantes : «Lorsque la difference de deux cas peut estre diminuée au dessous de toute grandeur donnée in datis ou dans ce qui est posé, il faut qu'elle se puisse trouver aussi diminuée au dessous de toute grandeur donnée in quaesitis ou dans ce qui en resulte, ou pour parler plus familierement : Lorsque les cas (ou ce qui est donné) s'approchent continuellement et se perdent enfin l'un dans l'autre, il faut que les suites ou evenemens (ou ce qui est demandé) le fassent aussi. » (GP III, 52). En rappelant l'article de 1687, l'ouvrage les Nouveaux Essais sur l'entendement humain donne d'autres formulations: «Rien ne se fait tout d'un coup, et c'est une de mes grandes maximes et des plus verifiées, que la nature ne fait jamais des sauts : ce que j'appellois la loi de la continuité... » (A VI, 6, 56) Ce principe permet de considérer la parabole, « comme une Ellipse dont un des foyers est infiniment eloigné », l'égalité « comme une inegalité infinement petite », le repos "comme une vistesse infinement petite, ou comme une tardité infinie» et donc la règle du repos « comme un cas particulier de la regle du mouvement » (GP III, 52). Sur le détail, voir entre autres F. Duchesneau, Leibniz et la Méthode de la Science, PUF, 1993, p. 311 ; et Idem., "Leibniz on the principle of the continuity ", Revue Internationale de Philosophie, Vol. 48, $\mathrm{n}^{\circ} 188$ (2), LEIBNIZ (1994), p. 141-160.

56. Selon, Sur le concept de loi de la nature, voir notamment André Charrak, Contingence et nécessité des lois de la nature. La philosophie seconde des Lumières, Paris, Vrin, 2006 ; Daston, Lorraine \& Michael Stolleis (éds.), Natural Law and Laws of Nature in Early Modern Europe : Jurisprudence, Theology, Moral and Natural Philosophy, Aldershot, Ashgate, 2008.

57. De necessitate eligendi optimum (1677?), A VI, 4, 1351; Specimen inventorum de admirandis naturae generalis arcanis (1688 ?) : «... Dieu agit comme un parfait Géomètre qui préfère les meilleures constructions des problèmes. » (A VI, 4, 1616., n. 1, trad. M, Fichant, Discours de métaphysique, Monadologie et autres textes, présentés, annotés et édités par M. F., Gallimard, coll. «Folio », Paris, 2004, p. 303). 
que Leibniz disait dans la lettre à Ch. Philippe de janvier 1680 que « de vouloir tout expliquer Mechaniquement en Physique, ce n'est pas un crime ny impieté, Dieu ayant tout fait selon les loix de mathematique, c'est à dire selon les verités eternelles qui sont l'objet de sagesse ${ }^{58}$. Or la thèse selon laquelle la souveraine sagesse a fait la nature en se réglant sur le principe de l'ordre général a deux conséquences majeures : en premier lieu, il n'y a pas d'irrégularités dans la nature; en second lieu, on peut réfuter les règles cartésiennes du mouvement en raison des incongruités qui en résultent et qui sont incompatibles avec le principe et partant avec la sagesse divine ${ }^{59}$.

Le deuxième point remarquable à cette époque en ce qui concerne la sagesse divine est, selon nous, une réflexion sur la notion complète ou parfaite individuelle et sur l'ordre général de l'univers considérés sous la raison de possibilité. Leibniz explicite le fondement théologique de l'idée de notion complète dans ce passage :

« Il est peu digne de Dieu de le concevoir (sous pretexte de maintenir sa liberté) à la façon de quelques Sociniens, et comme un homme, qui prend des resolutions selon les occurrences, et qui maintenant ne seroit plus libre de créer ce qu'il trouve bon [...]. Mais il faut plustost considerer que Dieu, choisissant, non pas un Adam vague, mais un tel Adam, dont une parfaite representation se trouve dans les idées de Dieu parmy les estres possibles, accompagné de telles circonstances individuelles... » (A II, 17).

Comme Dieu est le plus sage, il ne crée pas un être vague ou incomplet, mais un être complet parfaitement envisagé préalablement dans son entendement avec les circonstances et les suites. Et puisque toutes les volontés particulières sont parfaitement liées en Dieu, qui «penetre d'une seule veue » tout l'univers, " il faut considerer en Dieu une certaine volonté plus generale et plus comprehensive, qu'il a à l'egard de tout l'ordre de 1'univers ${ }^{60} \gg$ (A II, 18).

On constate ici que la perfection des ouvrages de Dieu, qui était décrite précédemment dans les termes de "l'horloge du monde » réglée au départ, est maintenant fondée sur la thèse de l'unicité du décret divin, laquelle se fonde, à son tour, sur la connaissance parfaite que la sagesse divine possède sur les choses possibles par la science de simple intelligence.

58. A, II, 1, 789. Rappelons d'ailleurs que l'image de Dieu-géomètre figurait déjà dans le paragraphe 59 de l'Hypothesis physica nova.

59. Cf. GP III, 53.

60. Leibniz continue un peu plus loin: «... plus on est sage, moins on a de volontés detachées et plus les veues et les volontés qu'on a sont comprehensives et liées. Et chaque volonté particuliere enferme un rapport à toutes les autres, afin qu'elles soyent les mieux concertées qu'il est possible. » (A II, 18). 


\section{L'hypothèse de l'harmonie préétablie et la sagesse divine}

Au cours des correspondances avec Arnaud qui suivent la discussion sur la notion complète, les nouvelles manières d'admirer la sagesse de Dieu commencent à ressortir notamment dans la théorie de l'organisme et dans l'hypothèse de concomitance qui marque le mieux la sagesse divine ${ }^{61}$. On peut mieux comprendre cette nouveauté si l'on rappelle certains articles du Discours de Métaphysique. Bien entendu, Leibniz y soulignait l'importance de la sagesse de Dieu. Tout en évoquant l'image de miroirs, cependant, il ne profitait pas assez de cette occasion pour l'admirer. En outre, pour ce qui est de la structure des animaux, il se contentait de dire que «Tous ceux qui voyent l'admirable structure des animaux se trouvent portés à reconnoistre la sagesse de l'auteur des choses $\gg{ }^{62}$.

Mais, c'est sans doute avec l'apparition du système de l'harmonie préétablie qui projette une nouvelle lumière sur la souveraine sagesse. Il s'agit de l'harmonie établie " par la sagesse infinie de l'auteur des choses » (À Basnage, GP III, 122). Il convient de remarquer que comparées avec les thèses précédentes, celles de ce système sont très souvent associées à la sagesse. Par ailleurs, bien que la considération de la sagesse elle-même reste importante comme c'est le cas dans le De rerum originatione radicali et dans les Essais de Théodicée, Leibniz se tourne vers les productions de la sagesse infinie plus qu'avant, en vue d'y trouver ses traces merveilleuses. Il ne s'agit plus seulement de trouver l'occasion de la connaître et de l'admirer dans l'optique et l'anatomie ainsi que dans les lois de la nature ${ }^{63}$, mais de le faire partout dans la nature. Ce changement se reflète dans la terminologie. D'une part, le terme de « merveille » attire particulièrement l'attention ${ }^{64}$. De

61. «... cette concomitance qui marque la sagesse admirable du createur bien plus que toute autre Hypothese ; on ne sçauroit disconvenir que cellecy ne soit au moins possible, et que Dieu ne soit assez grand ouvrier pour la pouvoir executer; apres quoy on jugera aisement que cette Hypothese est la plus probable, estant la plus simple, la plus belle et la plus intelligible... » (A II, 182. Cf. le Specimen inventorum de admirandis naturae generalis arcanis, 1688 ?) ; «... il n'y a point de parcelle de la matiere, dans la quelle ne se trouve un monde d'une infinité de creatures, tant organisées qu'amassées. Et sur tout que les ouvrages de Dieu sont infiniment plus grands, plus beaux, plus nombreux, et mieux ordonnés qu'on ne croit communement; et que la machine ou l'organisation, c'est à dire l'ordre, leur est comme essentiel jusque dans les moindres parties. Et qu'ainsi il n'y a point d'hypothese qui fasse mieux connoistre la sagesse de Dieu que la nostre, suivant la quelle il y a par tout des substances qui marquent sa perfection, et sont autant de miroirs mais differens de la beauté de l'univers, rien ne demeurant vuide, sterile, inculte et sans perception61» (A II, 259; GP II, 126).

62. A VI, 4, 1561. Cf. ces mots de F. Duchesneau, Leibniz: le vivant et l'organisme, Paris, Vrin, 2010, p. 87: « À mon avis, le tournant décisif menant à la version proprement leibnizienne des machines de la nature survient en corrélation avec la formulation de la "dynamique" proprement dite en 1690. Auparavant, la théorie ne s'éloigne guère de la version de type "malpigien" que nous avons vue à l'œuvre dans les inédits de 1680-1682... » comme Le corps de l'homme et de tout animal est une machine.

63. Cf. Tentamen anagogicum, GP VII, 279.

64. Cf. par exemple « merveilles de la nature » (e.g. GP III, 274, 279, 508). 
l'autre, Leibniz rappelle souvent l'attribut de sagesse avec celui de puissance parce que ces attributs correspondent aux causes finales et aux causes efficientes $^{65}$, et que l'harmonie requiert la sagesse qui l'envisage et la puissance qui l'exécute. De plus, ces attributs sont souvent qualifiés par l'adjectif $«$ infinies $»{ }^{66}$ pour souligner le fait que seul l'être infiniment parfait peut établir une telle harmonie merveilleuse. Ce fait fournit d'ailleurs une nouvelle occasion de prouver la nécessité de l'existence de Dieu ${ }^{67}$.

Les merveilles de la nature s'observent aussi bien dans la formation des animaux que dans la correspondance de l'âme avec le corps : "Comme pour expliquer cette merveille de la formation des animaux, je me servois d'une harmonie préétablie, c'est à dire, du même moyen dont je m'étois servi pour expliquer une autre merveille, qui est la Correspondance de l'ame avec le corps » (GP VI, 41). La formation des animaux doit être expliquée mécaniquement par la machine de la nature sans aucun besoin des natures plastiques, mais cette machine de la nature indestructible est elle-même prédisposée au départ par la sagesse de Dieu ${ }^{68}$. D'autre part, par sa sagesse et sa puissance infinies, «il a créé d'abord les ames et les corps de telle sorte, que chacun suivant ses propres loix se rencontre avec l'autre » (̇̀ Masham, GP III, 341).

Aussi Leibniz trouve-t-il de nouvelles manières d'utiliser la sagesse divine. Mais cela s'accompagne d'une critique quant à la mauvaise façon qu'a le système des causes occasionnelles d'utiliser Dieu. Il n'est pas bon de «l'employer toujours ex machina», dénonce-t-il (À Basnage De Bauval, 1695, A II, 3, 85, N 32). Il le précise dans le Système nouveau :

« Pour resoudre des problemes, il n'est pas assez d'employer la cause generale, et de faire venir ce qu'on appelle Deum ex machina. Car lorsque cela se fait sans qu'il y ait autre explication qui se puisse tirer de l'ordre des causes secondes, c'est proprement recourir au miracle. En Philosophie il faut tacher de rendre raison, en faisant connoistre de quelle façon les choses s'executent par la sagesse divine, conformement à la notion du sujet dont il s'agit » (Système nouveau, GP IV, 483).

Ajoutons un passage tiré de l'Addition à l'explication du système nouveau où Leibniz critique l'occasionalisme de François Lamy :

«Et il y a lieu de s'étonner, que des Philosophes excellens de ce temps peuvent concevoir que Dieu agit d'une maniere si arbitraire et si peu determinée (c'est à dire si destituée de raison), en establissant les loix de la nature soit pour les pensées soit pour les mouvemens. Ce n'est pas assez employer sa sagesse, qui est tousjours determinée à choisir le plus convenable » (Addition à l'explication du système nouveau, 1698-1700, GP IV, 576).

65. Cf. GP VI, 542.

66. Cf. par exemple GP VI, 555, GP IV, 586, GP III, 341.

67. Cf. par exemple GP IV, 578, GP VI, 555, GP IV, 504.

68. À Masham, GP III, 375. Cf. À Bourguet, GP III, 562. 
Dans l'optique de Leibniz, il faut bien employer Dieu, non pas seulement pour sa toute-puissance, mais aussi pour sa sagesse ${ }^{69}$, qui est toujours déterminée à choisir le plus convenable. Certes il sait que Malebranche reconnait l'excellence des ouvrages admirables de Dieu et la sagesse des voies ${ }^{70}$. Néanmoins, il n'est pas persuadé par les thèses malebranchistes. Dire que Dieu aurait pu faire un monde meilleur que le nôtre, c'est atteindre la perfection du monde. Même la thèse selon laquelle les voies de Dieu sont simples et fécondes, et donc sages, s'avère décevante pour Leibniz. Celui-ci objecte en effet aux occasionalistes que ces derniers ont recours à un miracle perpétuel peu convenable avec la philosophie et la souveraine sagesse ${ }^{71}$ et que leur Dieu agissant par sa « seule toute-puissance miraculeuse ${ }^{72}$ » ou par son « pouvoir arbitraire ${ }^{73}$ » ressemble à « un homme qui vit du jour au lendemain, ou à un ouvrier qui est tousjours occupé à raccommoder son ouvrage ${ }^{74} »$.

Nous avons vu que Leibniz revendique que son hypothèse de l'harmonie préétablie est la plus digne de la sagesse et de la puissance infinies de l'auteur de la nature. Selon lui, ces deux attributs sont marqués également par la thèse de la division actuelle à l'infini de la matière ${ }^{75}$, thèse qui constitue une condition de l'harmonie. La sagesse, dit-il, a fait que dans la nature il y ait partout des sous-divisions actuelles à l'infini pour que l'harmonie puisse se réaliser. Critiquant l'occasionalisme, il maintient que :

« ... il est convenable à la souveraine sagesse de donner à ses ouvrages qui sont dans l'ordre naturel, une nature ${ }^{76}$ où tout soit lié par des raisons, en sorte

69. D'ailleurs, la notion de la sagesse chez Malebranche est elle-même compliquée. Voir A. Robinet, Système et existence dans l'œuvre de Malebranche, Vrin, 1965, p. 22. Et même en possédant une bonne connaissance biologique de l'emboîtement des germes, Malebranche disait que « la beauté de l'univers ne consiste pas dans l'incorruptibilité de ses parties, mais dans la variété qui s'y trouve ; et ce grand ouvrage du monde ne serait pas si admirable sans cette vicissitude de choses que l'on y remarque » (Recherche de la vérité, III, II, X, Euvres complètes, tome 1, p. 482) et que «... il n'y a point d'instant dans la durée, comme il n'y a point d'atomes dans le corps; et de même que la plus petite partie de la matière se peut diviser à l'infini, comme il est facile de le démontrer» (Ibid., I, VIII, § II, p. 104 ; III, II, X, p. 480, p. 482). Sur la théorie de l'économie des animaux de l'époque et la sagesse infinie, cf. la remarque de J. Smith, Divine Machines, Princeton UP, 2011, p. 75.

70. Voir Malebranche à Leibniz, GP I, 358.

71. Cf. GP III, 341 et GP VI, 589.

72. GP VI, 589.

73. GP IV, 594.

74. GP IV, 586.

75. Une version précoce de cette thèse apparaissait, certes, déjà en 1676 avec la mention de la sagesse divine (De arcanis sublimium vel de summa rerum, 11 février 1676, A VI, 3, 474. Cf. Pacidius Philalethi, A VI, 3, 565). Mais elle est réinterprétée dans le cadre du système de l'harmonie prétablie.

76. Cette nature s'appelle aussi la force inhérente (vis insita) dans le De ipsa natura (GP IV, 507). Que lors de la réhabilitation des formes substantielles, Leibniz ait abandonné une sorte d'occasionalisme encore adoptée dans le De corporum concursu (1678), c'est ce que Paul Rateau a montré à la suite de Michel Fichant. Voir P. Rateau, «Les preuves 
que celuy qui seroit assez clairvoyant y pourroit lire l'avenir et le passé dans le present, et même l'estat de tout l'univers dans celuy de chaque partie. Ce qui ne se pourroit pourtant pas s'il n'y avoit partout des sousdivisions actuelles à l'infini, à fin que tout se puisse ressentir de tout autre. Et par ce moyen toute Ame ou Unité de substance, en representant originairement son corps, est representative de tout l'univers suivant sa portée $\mathrm{e}^{77} \gg$.

Ainsi, avec la plénitude, la division actuelle à l'infini de la matière conditionne la transmission du mouvement ${ }^{78}$, qui conditionne, quant à elle, la liaison spatio-temporelle de toutes choses et donc l'harmonie. Qui plus est, cette division infinie de la matière marque la sagesse divine non seulement comme condition de l'harmonie, mais en elle-même pour sa belle économie arrangée par la sagesse ${ }^{79}$. C'est la raison pour laquelle le corps organique d'un vivant ou la machine de la nature s'appelle aussi « Machine divine $^{80} »$. $\mathrm{Au}$ contraire, les atomes n'existent pas, parce que, quoique possibles absolument parlant ${ }^{81}$, ils sont incompatibles avec la sagesse divine, qui n'arrête pas la division sans raison et qui préfère l'ordre avec la variété et la diversité, etc ${ }^{82}$.

Pour ces raisons nous croyons que Leibniz trouve de nouvelles manières d'utiliser la sagesse divine dans l'hypothèse de l'harmonie préétablie et dans l'idée de machine de la nature. C'est pourquoi on peut sans doute dire que tout en cherchant toujours à considérer la sagesse divine en elle-même, il s'attache également à découvrir les merveilles dans les ouvrages de Dieu. En d'autres termes, ce n'est pas seulement l'harmonie considérée dans sa possibilité qui est ici thématisée, mais aussi l'harmonie effectivement établie, conditionnée par l'harmonie des possibles. Sans doute cela s'explique-t-il en partie par l'intention leibnizienne de faire un large usage de la sagesse divine.

L'usage de l'harmonie des possibles pour fonder l'harmonie des actuels s'observe également dans la controverse avec Clarke. Lisons d'abord le paragraphe 21 du cinquième écrit, qui oppose l'ordre des choses et la sagesse divine aux indiscernables ${ }^{83}$, conçus comme des termes abstraits et des notions incomplètes :

leibniziennes de l'existence de Dieu », p. 378; M. Fichant, Leibniz, La réforme de la dynamique. De corporum concursu et autres textes inédits, Springer, 1997, p. 134.

77. GP III, 383. Cf. GP VI, 626.

78. GP IV, 557.

79. À Masham, GP III, 340. Cf. À Sophie Charlotte, GP III, $344:$ «... elle [la matière] est l'Effet et l'emanation continuelle d'une souveraine intelligence... ».

80. Monadologie, § 64, GP VI, 618.

81. Cf. par exemple, GP VII, 394, 396, 405. Une possibilité métaphysique de l'existence d'une seule substance incompatible avec la sagesse est liée à la question de la compossibilité, que nous ne pouvons pas développer dans le présent article (cf. GP II, 307 ; GP II, 496 ; GP IV, 519 ; GP IV, 530).

82. Cf. par exemple À Lady Masham, GP III, 362 ; GP IV, 524 ; GP III, 507.

83. Nous ne pouvons pas détailler ici la relation entre la sagesse et le principe de l'identité des indiscernables. Cf. par exemple Gonzalo Rodriguez-Pereyra, Leibniz's Principle of Identity of Indiscernibles, Oxford, Oxford University Press, 2014, ch. 8. 
«Cette supposition de deux indiscernables, comme de deux portions de matiere qui conviennent parfaitement entre elles, paroist possible en termes abstraits; mais elle n'est point compatible avec l'ordre des choses, ny avec la sagesse Divine, où rien n'est admis sans raison. Le vulgaire s'imagine de telles choses, parce qu'il se contente de notions incompletes. Et c'est un des defauts des Atomistes » (GP VII, 394).

Leibniz poursuit dans le paragraphe suivant en affirmant que dans l'ordre des choses établi par la sagesse divine, " chaque portion de matière est actuellement sousdivisée en parties differement mues, et pas une ne ressemble entierement à l'autre » (GP VII, 394). Plus loin, dans le paragraphe 66, il appuie cette thèse sur une considération de la sagesse de Dieu, en un mot sur l'unicité de son décret :

«On dit que Dieu peut avoir des bonnes raisons pour placer deux cubes parfaitement egaux et semblables: et alors il faut bien (dit on) qu'il leur assigne leur places, quoyque tout soit parfaitement egal. Mais la chose ne doit point étre detachée de ces circonstances. Ce raisonnement consiste en notions incompletes. Les resolutions de Dieu ne sont jamais abstraites et imparfaites, comme si Dieu decernoit premierement à creer les deux cubes, et puis decernoit à part où les mettre...il n'y a qu'un seul decret pour l'univers tout entier... » (GP VII, 407).

Quelle conséquence peut-on en tirer pour la «métaphysique »? Il nous semble qu'à mesure que l'objectif d'utiliser la sagesse se satisfait, la nature n'est plus considérée simplement comme domaine au-delà duquel se situe le principe métaphysique, mais plutôt comme les ouvrages admirables du principe métaphysique qui est Dieu. Autrement dit, la nature n'est plus simplement ce qui doit être expliqué par les causes finales comme dans le cas de l'optique, mais elle est pensée également comme ce qui nous montre partout les traces de la sagesse de son auteur. D'où résulte la juxtaposition de la nature et de la sagesse ${ }^{84}$. C'est sans doute pour cela que Leibniz thématise non seulement la thèse de la dépendance de la physique envers la métaphysique, mais aussi le passage de la mathématique à la physique. En d'autres termes, l'opposition se voit non seulement entre physique et métaphysique ou entre mathématique et métaphysique, mais aussi entre mathématique et physique fondée sur le principe métaphysique. À cet égard, il convient de citer le passage suivant :

« D'autre part, pour passer des idées de la géométrie aux réalités de la physique, je déclare que la matière est actuellement divisée en parties plus petites que n'importe quelle partie donnée, c'est-à-dire qu'il n'y a aucune partie qui ne soit actuellement subdivisée en d'autres exerçant d'autres mouvements. Cela est postulé par la nature de la matière et du mouvement, et par

84. Voir par exemple, GP VII, 393, 563. 
l'assemblage complet des choses, pour des raison Physiques, Mathématiques et Métaphysiques $»{ }^{85}$.

Ainsi, la physique qui traite des choses réelles dans la nature, entendues comme ouvrages de la sagesse divine, se distingue nettement de la mathématique qui porte sur les notions abstraites et incomplètes. Il n'en demeure pas moins que les phénomènes de la nature observent les règles de la mathématique, comme en témoigne clairement une réponse à Bayle datée de 1702 :

«... neantmoins les phenomenes actuels de la nature sont menagés et doivent l'estre de telle sorte, qu'il ne se rencontre jamais rien, où la loy de la continuité (que j'ay introduite, et dont j'ay fait la premiere mention dans les Nouvelles de la Republique des Lettres de M. Bayle) et toutes les autres règles les plus exactes des Mathematiques soient violées » (GP IV, 569).

Dans la «Lettre de M. L. sur un principe general... » à laquelle Leibniz renvoie, nous avons vu que, si le principe de l'ordre général est applicable de la mathématique à la physique, c'est «par ce que la souveraine sagesse [...] agit en parfait geometre ${ }^{86} \gg$. Certes la nature diffère du domaine de la mathématique dans la mesure où celle-là est la production admirable de la sagesse infinie, alors que celle-ci traite des objets abstraits et incomplets. Néanmoins, puisque la sagesse a créé la nature en se réglant sur les règles mathématiques, la nature les observe. En ce sens, dans la «variété infiniment infinie » de la nature, Leibniz déclare qu' ' il y a de l'Harmonie, de la Géometrie, de la Metaphysique, et pour parler ainsi de la morale par tout ${ }^{87} »$.

\section{Conclusion : la sagesse divine comme fin de la philosophie}

Dans cet article nous avons examiné les diverses fonctions que Leibniz assigne à la notion de la sagesse divine dans son œuvre, en particulier dans le contexte physique et métaphysique. La sagesse divine, qui n'était pas inséparablement liée à la meilleure des séries possibles ni à la " métaphysique» au sens strict du terme, va s'unir avec elles et caractériser cette nouvelle métaphysique dominante comme métaphysique de la sagesse

85. À des Bosses, 11 mars 1706, GP VII, 305, tr. par C. Frémont, L'être et la relation, Paris, Vrin, 1999, p. 95. Cf. GP VII, 563 et 355-356.

86. GP III, 52. Ce que la lettre à Varignon du 16 octobre 1707 exprime en termes d'harmonie entre physique et géométrie : "la physique harmonise constamment avec la géométrie » (L. Prenant, 1972, 376).

87. À Bossuet, 18 Avril 1692, A II, 2, 515. 
divine. Leibniz commence à se servir utilement de cette dernière en physique. Dans la seconde moitié des années 1680 , il va réaliser le même dessein au travers du principe de l'ordre général. En outre, à l'occasion du débat autour de la notion complète individuelle, il développe un argument détaillé sur certaines propriétés de la sagesse de Dieu, notamment sur l'unicité de son décret, ce qui fonde l'image de l'horloge du monde. Ensuite, au moment de la naissance de l'hypothèse de l'harmonie préétablie ainsi que de l'idée de machine de la nature, il s'engage à ouvrir de nouvelles voies d'utiliser la sagesse. La tendance à reconnaître les merveilles partout dans la nature qui est l'ouvrage admirable de Dieu se trouve renforcée. Sans parler des substances simples, des âmes et des esprits, même une parcelle de la matière contient une infinité de créatures et représente tout l'univers de son point de vue. Il s'agit d'une chose intelligible, à laquelle ni nos sens ni notre imagination ne peuvent accéder, et qui requiert et nécessite une analyse poussée jusqu'à la dernière raison et par conséquent la considération de la sagesse divine. Dans la variété infiniment infinie de la nature, on constate l'harmonie entre géométrie, métaphysique et morale. C'est dans cet ordre des choses établi par la sagesse et non pas dans les choses possibles métaphysiquement parlant que la métaphysique réelle trouve ses véritables objets actuels.

Notre connaissance de la sagesse divine ne peut pas et ne doit pas aller aux jusqu'aux détails : "c'est assez de le sçavoir sans le comprendre. Et c'est icy qu'il est temps de reconnoistre altitudinem divitiarum, la profondeur et l'abyme de la divine sagesse, sans chercher un detail qui enveloppe des considerations infinies ${ }^{88}$. Mais on en est capable de manière générale. À Lady Masham, qui se demande «si nous pouvons determiner par la consideration de cette sagesse infinie, quelles en doivent estre les operations » (GP III, 362), Leibniz répond que « nous le pouvons en general et en excluant souvent ce qui n'y est point conforme, mais non pas en detail» (Ibid.). En effet, « on ne sçauroit attribuer trop de reflexions à cette sagesse infinie, et il n'y a aucune matiere où il y aye moins d'erreur à craindre, tandis qu'on ne fait qu'affirmer, et pourveu qu'on se garde icy des propositions negatives, qui limitent les desseins de Dieu » (A VI, 4, 1560).

C'est seulement sous cette condition que l'on est autorisé à faire la recherche des causes finales que Spinoza a dénoncées comme préjugé et que Descartes a retranchées de la philosophie naturelle "sous ce pretexte adroit que nous ne sommes pas capables de sçavoir les fins de Dieu » (A II, 1, 778). Connaître la sagesse et la puissance divines proprement, c'est, loin d'abuser de notre pouvoir, bel et bien reconnaître la grandeur de Dieu contre $l^{\prime}$ « anthropomorphisme ${ }^{89}$. Faire connaître et admirer la sagesse divine, c'est le but de la philosophie, voire cela doit être «le plus grand but de

88. A VI, 4, 1577. Cf. Essais de théodicée (II, § 434), GP VI, 188.

89. Cf. Causa Dei, § 2, GP VI, 439. 
la philosophie », pour reprendre les paroles de Leibniz ${ }^{90}$. S'agit-il d'une exagération? Nous ne le pensons pas. La connaissance de la sagesse qui est la plus parfaite tant métaphysiquement que moralement est essentielle au projet leibnizien de réconcilier raison et piété, puisqu'elle nous invite à honorer et aimer Dieu plus que toutes autres choses ${ }^{91}$. Elle correspond, d'ailleurs, au but de la création divine. En théologien, Leibniz soutient en effet que :

«Lors de la création, Dieu s'est proposé de communiquer sa gloire, pour que sa sagesse soit connue aussi par les autres, de créer les créatures à son image, et de constituer toutes choses de telle sorte qu'elles soient les plus parfaites pour les créatures rationnelles ou que plus celles-là pénètrent dans l'intérieur des choses, plus elles leur plairont $»^{92}$.

C'est pourquoi on pourrait dire que la philosophie de Leibniz, qui s'attache à faire connaître et admirer la sagesse divine, est son effort incessant de satisfaire aux fins de Dieu.

En déplaçant la considération de la sagesse divine de la théologie vers la physique, nous avons retracé ses évolutions dans l'œuvre de Leibniz. En fin de compte, ce changement de regard nous permet d'unifier le concept de sagesse divine comme fin de la philosophie.

90. « La consideration de la sagesse divine dans l'ordre des choses, qui à mon avis doit estre le plus grand but de la philosophie » (À Nicaise, GP III, 562) ; "Je voudrois qu'on s'attachât à faire connoistre la sagesse de Dieu par la physique et mathematique, en decouvrant de plus en plus les merveilles de la nature. C'est là le vray moyen de convaincre les profanes, et doit estre le but de la philosophie» (GP III, 279. Cf. GP III, 274) ; «C'est sanctifier la philosophie, que de faire couler ses ruisseaux de la fontaine des attributs de Dieu. Bien loin d'exclure les causes finales et la consideration d'un estre agissant avec sagesse, c'est de là qu'il faut tout deduire en physique » (GP III, 54).

91. «... l'essentiel de la pieté est non seulement de le craindre, mais encor de l'aimer sur toutes choses, ce qui ne se peut sans qu'on en connaisse les perfections capables d'exciter l'amour qu'il merite, et qui fait la felicité de ceux qui l'aiment » (Théodicée, § 6, GP VI, 106). 92. De libertate a necessitate in eligendo, A VI, 4, 1453 (été 1680 à été 1684 (?)), notre traduction. 